\title{
O Programa Choosing Wisely Portugal: Escolhas Criteriosas em Saúde Choosing Wisely Portugal: Wise Health Decisions
}

\author{
Inês Urmal' (https://orcid.org/0000-0001-6817-1445), Ana Mafalda Abrantes ${ }^{2,3}$ (https://orcid.org/0000-0002-1295- \\ 9679), Martim Trovão Bastos ${ }^{4,5}$ (https://orcid.org/0000-0002-8702-1183), Miguel Bigotte Vieira ${ }^{5,6}$ (https://orcid. \\ org/0000-0003-0528-2716)
}

Palavras-chave: Comunicação em Saúde; Literacia em Saúde; Qualidade dos Cuidados de Saúde.

Keywords: Health Communication; Health Literacy; Quality of Health Care.

O artigo de Watts et al, publicado recentemente nesta revista, aborda um problema atual e transversal aos cuidados de saúde em Portugal. ${ }^{1}$ A Organização Mundial da Saúde define literacia em saúde como o conjunto de "competências cognitivas e sociais e a capacidade da pessoa para aceder, compreender e utilizar informação por forma a promover e a manter uma boa saúde”. ${ }^{2}$ Este conceito implica não só o conhecimento, mas também a capacidade de utilizar a informação para a tomada de decisões fundamentadas no que diz respeito aos cuidados de saúde, prevenção de doença e promoção da saúde individual e da comunidade., ${ }^{2,3}$ De acordo com Watts et al, após aplicação de um questionário dirigido a doentes internados numa enfermaria de Medicina Interna num hospital terciário da Grande Lisboa, 68,8\% dos inquiridos apresentavam grau problemático ou inadequado de literacia em saúde. ${ }^{1}$ Este resultado vai de encontro ao obtido no inquérito sobre Literacia em Saúde em Portugal (ILS-PT), em 2016, o qual salienta que $38,1 \%$ da população portuguesa apresenta um nível problemático de literacia em saúde, acima da média europeia $(35,2 \%) .^{3}$ Segundo os autores, parece ainda haver um impacto negativo da idade na literacia em saúde, sem aparente relação com o grau de escolaridade do doente. Tal facto poderá conduzir a uma utilização inadequada dos serviços de saúde, bem como limitada comunicação

\footnotetext{
${ }^{1}$ Unidade Funcional de Medicina 2.1, Hospital de Santo António dos Capuchos, Centro Hospitalar e Universitário de Lisboa Central, Lisboa, Portugal

${ }^{2}$ Serviço de Medicina 2, Hospital de Santa Maria, Centro Hospitalar de Lisboa Norte, Lisboa, Portugal

${ }^{3}$ Instituto de Semiótica Clínica, Faculdade de Medicina da

Universidade de Lisboa, Lisboa, Portugal

${ }^{4}$ Serviço de Medicina 3, Hospital Prof. Doutor Fernando Fonseca, Amadora, Portugal

${ }^{5}$ Instituto de Saúde Baseada na Evidência, Universidade de Lisboa, Lisboa, Portugal

${ }^{6}$ Serviço de Nefrologia, Hospital Curry Cabral, Centro Hospitalar e Universitário de Lisboa Central, Lisboa, Portugal.
}

DOI:10.24950/CE/172/20/4/2020 entre médico e doente, com consequente compromisso na adesão a métodos de diagnóstico e terapêutica, culminando em gastos excessivos em saúde. ${ }^{2-4}$ No artigo de referência, são apresentadas sugestões dos inquiridos para o aumento da literacia em saúde, designadamente a existência de veículos de informação acessível, com o envolvimento dos profissionais de saúde na prestação de esclarecimentos em áreas como promoção de saúde e prevenção de doença, bem como no fornecimento de materiais explicativos elaborados pelas autoridades em saúde. Destaca-se ainda a necessidade de utilização de linguagem simples e eficaz, de forma a optimizar a comunicação. ${ }^{1}$ Felicitamos os autores pela avaliação desta preocupante realidade na população portuguesa e salientamos a implementação do programa Choosing Wisely Portugal - Escolhas Criteriosas em Saúde, em 2018. ${ }^{5}$ Este programa foi fundado em 2012 pelo American Board of Internal Medicine e foi posteriormente adaptado e implementado em mais de 20 países. Tem como objetivo a promoção de escolhas em Saúde baseadas na melhor evidência científica disponível, reduzindo o número de intervenções desnecessárias, sem eficácia comprovada e/ou com uma relação risco-benefício desfavorável. Sendo um projeto da Ordem dos Médicos, trata-se de um programa global de Educação em Saúde, dirigido quer à população em geral, quer aos profissionais de saúde de todas as áreas e graus de diferenciação. Atualmente, conta com a colaboração de 30 Colégios da Especialidade, que emitiram já cerca de 150 recomendações, disponíveis para consulta online em https://ordemdosmedicos.pt/cwp-escolhasaude/. Destacamos, em particular, o apoio do colégio da especialidade de Medicina Interna, que já emitiu recomendações relativas a prescrição racional de fármacos e utilização adequada de meios complementares de diagnóstico e terapêutica. Em conjunto com a Sociedade Portuguesa de Medicina Interna, o programa é apoiado também por cerca de 30 outras sociedades científicas e 20 associações de doentes. Considerando essencial a promoção do programa junto da sociedade civil, ao longo dos últimos anos tem vindo a ser consolidada a presença do Choosing Wisely Portugal junto dos media e redes sociais. Neste sentido, e dando continuidade à divulgação do programa, encontram-se planeadas atividades de divulgação do programa dirigidas a estudantes de medicina, bem como utilizadores e prestadores de serviços em cuidados de saúde primários e serviços hospitalares. 
CARTAS AO EDITOR

\section{Responsabilidades Éticas}

Conflitos de Interesse: Os autores declaram a inexistência de conflitos de interesse na realização do presente trabalho.

Fontes de Financiamento: Não existiram fontes externas de financiamento para a realização deste artigo.

Proveniência e Revisão por Pares: Não comissionado; revisão externa por pares.

\section{Ethical Disclosures}

Conflicts of interest: The authors have no conflicts of interest to declare. Financing Support: This work has not received any contribution, grant or scholarship.

Provenance and Peer Review: Not commissioned; externally peer reviewed.

(c) Autor (es) (ou seu (s) empregador (es)) e Revista SPMI 2020. Reutilização permitida de acordo com CC BY-NC. Nenhuma reutilização comercial. (C) Author(s) (or their employer(s)) and SPMl Journal 2020. Re-use permitted under CC BY-NC. No commercial re-use.

\section{Correspondence / Correspondência:}

Inês Urmal - ines.urmal@chlc.min-saude.pt

Unidade Funcional de Medicina 2.1, Centro Hospitalar e Universitário de
Lisboa Central, Hospital de Santo António dos Capuchos, Lisboa, Portugal Alameda de Santo António dos Capuchos, 1169-050 Lisboa

Received / Recebido: 23/08/2020

Accepted / Aceite: 23/08/2020

Publicado / Published: 18 de Dezembro de 2020

\section{REFERÊNCIAS}

1. Watts-Soares A, Maia M, Vistonti V, Fernandes A, Espírito Santo J, Oliveira I, et al. Literacia em Saúde nos Doentes Hospitalizados num Serviço de Medicina Interna. Rev Soc Port Med Interna. 2020; 27: 124-30.

2. Kickbusch I. Navigating Health: The Role of Health Literacy. London: Alliance For Health And The Future, International Longevity Centre; 2005. [consultado a 05/08/2020] Disponível em: https://ilcuk.org.uk/navigating-health-the-role-of-health-literacy/.

3. Plano De Ação, Literacia Em Saúde Portugal 2019-2021, Direção de Serviços de Prevenção da Doença e Promoção da Saúde (DSPDPS) Divisão de Literacia, Saúde e Bem-Estar, Direção-Geral da Saúde, 2019. [consultado a 05/08/2020] Disponível em: https://www.dgs.pt/documentos-e-publicacoes/plano-de-acao-para-a-literacia-em-saude-2019-2021-pdf. aspx.

4. Pedro AR, Amaral $\mathrm{O}$, Escoval A. Literacia em saúde, dos dados à acção: tradução, validação e aplicaçãodo European Health Literacy Surveyem Portugal. Rev Port Saúde Pública. 2016; 34: 259-75

5. Bigotte Vieira M, Ferreira Dos Santos G, Carvalho CR, et al. Choosing Wisely Portugal - Escolhas Criteriosas em Saúde. Acta Med Port. 2018; 31: $521-3$ 